\title{
Early detection of colorectal adenocarcinoma: a clinical decision support tool based on plasma porphyrin accumulation and risk factors
}

Manuela Lualdi ${ }^{1 *}$ (D) Adalberto Cavalleri ${ }^{2}$, Luigi Battaglia ${ }^{3}$, Ambrogio Colombo $^{4}$, Giulia Garrone ${ }^{2}$, Daniele Morelli ${ }^{5}$, Emanuele Pignoli ${ }^{1}$, Elisa Sottotetti ${ }^{6}$ and Ermanno Leo $^{3}$

\begin{abstract}
Background: An increase in naturally-occurring porphyrins has been described in the blood of subjects bearing different kinds of tumors, including colorectal, and this is probably related to a systemic alteration of heme metabolism induced by tumor cells. The aim of our study was to develop an artificial neural network (ANN) classifier for early detection of colorectal adenocarcinoma based on plasma porphyrin accumulation and risk factors.

Methods: We measured the endogenous fluorescence of blood plasma in 100 colorectal adenocarcinoma patients and 112 controls using a conventional spectrofluorometer. Height, weight, personal and family medical history, use of alcohol, red meat, vegetables and tobacco were all recorded. An ANN model was built up from demographic data and from the integral of the fluorescence emission peak in the range 610-650 nm. We used the Receiver Operating Characteristic (ROC) curve to assess performance in distinguishing colorectal adenocarcinoma patients and controls. A liquid chromatography-high resolution mass spectrometry (LC-HRMS) analytical method was employed to identify the agents responsible for native fluorescence.

Results: The fluorescence analysis indicated that the integral of the fluorescence emission peak in the range 610$650 \mathrm{~nm}$ was significantly higher in colorectal adenocarcinoma patients than controls $(p<0.0001)$ and was weakly correlated with the TNM staging (Spearman's rho $=0.224, p=0.011$ ). LC-HRMS measurements showed that the agents responsible for the fluorescence emission were mainly protoporphyrin-IX (PpIX) and coproporphyrin-I (Cpl). The overall accuracy of our ANN model was $88 \%$ ( $87 \%$ sensitivity and $90 \%$ specificity) with an area under the ROC curve of 0.83 .

Conclusions: These results confirm that tumor cells accumulate a diagnostic level of endogenous porphyrin compounds and suggest that plasma porphyrin concentrations, indirectly measured through fluorescence analysis, may be useful, together with risk factors, as a clinical decision support tool for the early detection of colorectal adenocarcinoma. Our future efforts will be aimed at examining how plasma porphyrin accumulation correlates with survival and response to therapy.
\end{abstract}

Keywords: Colorectal cancer, adenocarcinoma, tumor marker, native fluorescence, Protoporphyrin IX, Coproporphyrin I

\footnotetext{
* Correspondence: manuela.lualdi@istitutotumori.mi.it

${ }^{1}$ Medical Physics Unit, Fondazione IRCCS Istituto Nazionale dei Tumori, Via

Venezian 1, 20133 Milan, Italy

Full list of author information is available at the end of the article
}

(c) The Author(s). 2018 Open Access This article is distributed under the terms of the Creative Commons Attribution 4.0 International License (http://creativecommons.org/licenses/by/4.0/), which permits unrestricted use, distribution, and reproduction in any medium, provided you give appropriate credit to the original author(s) and the source, provide a link to the Creative Commons license, and indicate if changes were made. The Creative Commons Public Domain Dedication waiver (http://creativecommons.org/publicdomain/zero/1.0/) applies to the data made available in this article, unless otherwise stated. 


\section{Background}

About $90 \%$ of people whose colorectal cancer (CRC) is diagnosed before it has spread to nearby lymph nodes or organs (localized stage) survive more than five years after diagnosis. However, only $14 \%$ of those whose cancer has spread to distant parts of the body (distant stage) survive five years [1]. CRC progresses slowly from detectable and curable precancerous lesions, so diagnosis at an early stage and removal of clinically significant adenomas aims to reduce the incidence of advanced tumors and hence mortality. Although there is proven evidence that screening reduces the incidence of $\mathrm{CRC}[2,3]$, the widespread diffusion of the most effective examination techniques such as colonoscopy and sigmoidoscopy is limited by the availability of resources and by low compliance. Acceptable and inexpensive filter tests should boost the numbers of people who undergo regular screening and should select for colonoscopy or sigmoidoscopy those who are most likely to benefit.

Currently the fecal immunochemical test (FIT) is considered the screening test of choice for CRC: it shows greater sensitivity than the guaiac-based fecal occult blood test (gFOBT) $[4,5]$, is more specific and less expensive than the FIT-DNA test $[4,6]$ and has higher specificity and a better positive likelihood ratio than fecal M2-type pyruvate kinase [7]. However, blood tests are likely to be more acceptable than stool tests in population-based screening [8]. Recently several approaches based on antibody signature have been developed for early detection of CRC, but further validation studies are required before they can be proposed in clinical practice [9].

There are several biomolecules which, when excited at suitable wavelengths, give fluorescence emission over a wide spectral range. As a pathological condition develops in tissue, changes occur in biochemical, physicochemical and histological properties at the cellular and tissue levels and the fluorescence emission spectrum may show changes, facilitating the distinction between normal and malignant tissue. This finding has been already applied in the early detection of breast, cervix, colorectal and oral cancer [10-13].

In previous works $[14,15]$, we investigated the possible use of the endogenous fluorescence of blood plasma for the early detection of colorectal adenocarcinoma, which accounts for $96 \%$ of all CRC cases [4]. In these studies, the only parameter used to discriminate between colorectal adenocarcinoma patients and their control counterparts was the intensity of fluorescence at $623 \mathrm{~nm}$, whose overall accuracy in distinguishing the two populations was $73 \%$ ( $80 \%$ sensitivity and $50 \%$ specificity). Subsequent investigations have suggested extending the fluorescence signal analysis over a wider spectral range and using the risk factors for this pathology together with the results of fluorescence analysis to boost the diagnostic power of the developing marker.
In this pilot study we used the integral of the fluorescence emission peak in the range 610-650 nm, hereafter referred to as IF-INT, and the demographic data of the subjects enrolled to train an artificial neural network (ANN) multiparametric test for colorectal adenocarcinoma. The primary objective was to verify the performance of the classifier for distinguishing patients with colorectal adenocarcinoma from control subjects. The second endpoint was to identify the agents responsible for the fluorescence signal by liquid chromatography-high resolution mass spectrometry (LC-HRMS).

\section{Methods \\ Participants}

Between January 2013 and December 2014, we consecutively recruited all colorectal adenocarcinoma patients who accessed the Colon-Rectal Surgery Unit of the Fondazione IRCCS Istituto Nazionale Tumori in Milan, Italy, and who agreed to participate in the study. Patients were eligible if they were more than 18 years old, had never had other tumors, had no concomitant non-tumor gastrointestinal diseases such as Crohn's disease and diverticulitis, had not undergone chemotherapy in the six months prior to admission and had a histopathologic diagnosis of colorectal adenocarcinoma. A blood sample was taken before surgery from all patients meeting the selection criteria. In the same period, we recruited controls among the patients who entered the Endoscopy Unit of our Institute to undergo colonoscopy for family history, following a positive outcome of FIT or gFoBT or for a bleeding episode that required immediate diagnostic assessment. Subjects were eligible if they were more than 18 years old, had never had cancer, did not have concomitant gastrointestinal diseases such as Crohn's disease and diverticulitis, and colonoscopy confirmed the absence of colorectal carcinoma, adenomas or inherited syndromes. A blood sample was taken before colonoscopy from all the patients meeting the selection criteria.

All the subjects enrolled gave written informed consent to participate and completed a questionnaire to record information on height and weight, alcohol intake (none, $<1$ and $\geq 1 \mathrm{drink} /$ day), red meat (none, 1-2, 3-4 and $>4$ portions/week) and vegetables (none, 1 and $>1$ portion/day), smoking status (never, former or current smoker) and family history of CRC (yes or no, up to second-degree relatives).

The Ethics Committee of our Institute approved this study protocol before subjects were enrolled.

\section{Fluorescence measurements}

Blood samples were collected in lithium-heparin tubes, centrifuged and the supernatant was collected. All supernatants were stored at $-20^{\circ} \mathrm{C}$ until analysis. The fluorescence 
analysis method has been described in detail previously [14]. Briefly, fluorescence was measured in plasma samples with a conventional spectrofluorimeter (Model F-3000, Hitachi Ltd. Tokyo, Japan), selecting an excitation wavelength of $405 \mathrm{~nm}$ and recording the fluorescence emission spectra in the range $430-700 \mathrm{~nm}$. In view of the broad variability in the fluorescence intensity from sample to sample, each fluorescence spectrum was normalized by dividing the fluorescence intensity at each wavelength by the maximum intensity of the spectrum and the IF-INT was finally calculated. The non-parametric Mann-Whitney test for unpaired data was employed to assess differences in the mean IF-INT between colorectal adenocarcinoma patients and control subjects. The Spearman correlation analysis was performed to evaluate the correlation between IF-INT and the TNM-UICC classification of the disease [16]. A $p$ value $<0.05$ was taken to indicate statistical significance.

The predictive performance of IF-INT was assessed from the area under the receiver operating characteristic curve (AUROC). Sensitivity, specificity, negative predictive value (NPV) and positive predictive value (PPV) were also calculated.

\section{Artificial neural network}

Artificial neural networks are ideal for modeling non-linear relationships between a set of predictors or input variables and one or more responses or output variables. The action of an ANN is defined by the neurons of each layer, which are the basic computational units of the network, and by the connections between the layers, with their weights. Among all the possible network architectures, the feed-forward neural network with back-propagation training has been widely adopted for realistic nonlinear multiple regression in different medical fields [17]. In a feed-forward network, each neuron is connected to all the neurons of the previous and subsequent layers, with no connections between neurons on the same layer [18].

In order to develop a classifier to distinguish colorectal adenocarcinoma patients from controls, we built up a feed-forward ANN model using MATLAB software (The Mathworks Inc., Natick, MA). Seven variables were considered as inputs for the network: IF-INT, body mass index (BMI), calculated by dividing the weight in kilograms by the square of the height in meters, alcohol consumption, red meat intake, vegetables intake, smoking status and family history of CRC. A three-layer structure was used with only one hidden layer; during network instruction, the number of hidden neurons was varied between 1 and 6 . The output of our model was between 0 and 1 .

The first phase of model development is the training procedure on a defined set of input variables with known output data. The overall population was randomly divided into three subsets, training, validation and test sets, and the back-propagation algorithm with the early stopping procedure was applied; the leave-one-out cross-validation tool was adopted in order to avoid overfitting the data [19]. Multiple runs of the ANN model were done, with the number of hidden neurons ranging between 1 and 6 , with random choices for the weights and biases at each cycle and changing the relative composition of the training, validation and test sets. The moment of stopping the training procedure and the final topology of the network were both decided by minimizing on the validation set the mean square error (MSE), which is the measure of how the predicted and actual data differ. Finally, we examined a test set of data completely unknown to the network and evaluated the predictive performance of the ANN model through ROC analysis. Sensitivity, specificity, NPV and PPV were calculated by imposing a cut-off of 0.60 on the ANN output.

After optimizing the ANN model, the relative importance (RI\%) of the input variables was assessed through the most squares method [20] to evaluate the role of each predictor in the prediction process.

\section{Qualitative mass-spectrometric analysis}

Several studies suggest that tumor cells are able to produce porphyrins naturally or after administering their precursor [13, 21-23] and that porphyrin compounds are responsible for plasma red fluorescence $[24,25]$. On this basis, we assumed that the difference between the blood fluorescence spectra of colorectal adenocarcinoma patients and control subjects was due to endogenous porphyrins accumulated in cancer cells as a result of a systemic alteration of heme metabolism, and then pumped out to plasma. To identify the molecules responsible for the fluorescence signal, we developed an LC-HRMS method to determine the mass-to-charge ratio $(\mathrm{M} / \mathrm{Z})$ with $1 \mathrm{ppm}$ error for the substances isolated from the plasma samples. This information, together with the retention time (RT), serves to determine the nature of the substances themselves.

In detail, $1000 \mu \mathrm{L}$ of ethyl acetate:acetic acid $(3: 1 \mathrm{v} / \mathrm{v})$ were added to $400 \mu \mathrm{L}$ of plasma; after vortexing and centrifugation at 17000 relative centrifugal force (RCF-g) for $20 \mathrm{~min}$ at $15^{\circ} \mathrm{C}$, the supernatant was transferred into a polypropylene tube. The organic layer was evaporated in a stream of nitrogen at $40{ }^{\circ} \mathrm{C}$. The dry residue of plasma samples was reconstituted with $100 \mu \mathrm{L}$ water: aceto- nitrile:acetic acid $2: 1: 1 \mathrm{v} / \mathrm{v} / \mathrm{v}$ and $15 \mu \mathrm{L}$ were injected into the LC-HRMS system.

Liquid chromatography separation was done with a Dionex Ultimate 3000 RSLC (Thermo Fisher Scientific, Waltham, MA) equipped with an XBridge BEH300-C18 $3.5 \mu \mathrm{m}, 150 \times 2.1 \mathrm{~mm}$ column (Waters Corporation, Milford, MA). A binary mobile phase and gradient elution 
were used at a flow rate of $350 \mu \mathrm{L} / \mathrm{min}$. Mobile phases were: $\mathrm{A}, \mathrm{H}_{2} \mathrm{O}$ with $10 \%$ acetic acid and $\mathrm{B}$, acetonitrile with $10 \%$ acetic acid. In the first $6 \mathrm{~min}$, phase $\mathrm{B}$ was raised from 40 to $98 \%$; for the next 2 min phase B was kept at 98\%; finally, reconditioning to the initial phase composition was scheduled in the last $5 \mathrm{~min}$.

An Orbitrap Elite high-resolution mass spectrometer (Thermo Fisher Scientific, Waltham, MA) was used as detector, working in electro-spray ionization mode in positive polarity: spray voltage was $4.5 \mathrm{kV}$; sheath and auxiliary gas (nitrogen) pressure were 40 and 15 a.u. respectively; the vaporizer temperature was $320{ }^{\circ} \mathrm{C}$; the capillary temperature was $350{ }^{\circ} \mathrm{C}$; s-lens was $69 \mathrm{~V} . \mathrm{M} / \mathrm{Z}$ values were acquired in full scan (FS) mode (mass range 250-1300 Da); resolving power was set to 120.000 full width at half maximum (FWHM) to permit exact mass extraction of molecular ions from the FS spectra; chromatographic RTs were used to ensure reliable compound identification.

Five analytes were selected that are involved in heme biosynthesis and have characteristic fluorescence emission in the range 610-650 nm: protoporphyrin-IX (PpIX), coproporphyrin-I (CpI), iron protoporphyrin IX (FePpIX), biliverdin (Bv) and protoporphyrin IX dimethyl ester (dmPpIX) (Sigma Aldrich, St. Louis, MO). These analytes, each at a concentration of $1 \mathrm{mg} / \mathrm{ml}$, were used to prepare a standard solution. Twenty plasma samples with and 20 without evidence of native fluorescence, and the porphyrin standard solution, were processed with the analytical method.

\section{Results}

\section{Participants}

From January 2013 to December 2014, 135 patients who accessed the colorectal surgery unit of our Institute were consecutively enrolled. Fifteen were excluded for missing information; 12 were non-eligible because they had received chemotherapy in the six months prior to blood sampling, five patients because their blood sample was hemolyzed and therefore not analyzable; three were excluded after surgery as they were found not to have adenocarcinoma. The colorectal adenocarcinoma patients were classified postoperatively according to the TNM-UICC classification based on clinical and pathological findings. Seven patients were stage 0, 31 stage I, 29 stage II, 25 stage III, and 8 stage IV. In the same period, 157 patients who accessed the endoscopy unit of our Institute were recruited. Thirty-one were excluded as the colonoscopy and the histological exam confirmed the presence of an adenoma, familial adenomatous polyposis or Crohn's disease; 14 patients were excluded for missing information.

The main demographic characteristics of the study population are summarized in Table 1. Patients and controls were divided into four groups according to their BMI: underweight $\left(<18.5 \mathrm{Kg} / \mathrm{m}^{2}\right)$, normal $(18.50-24.99)$, overweight (25.00-29.99) and obese $(\geq 30.00)$ [26]. Colorectal adenocarcinoma patients were older than controls, with similar mean ages for women and men in both groups. Distributions of BMI, smoking, alcohol and meat consumption by sex indicated a healthier lifestyle for women than men. In addition, differences in BMI and alcohol and meat consumption between colorectal adenocarcinoma patients and control groups were more marked for men than women. Among men, colorectal adenocarcinoma patients were less frequently normal weight and more frequently overweight, had higher alcohol and red meat intake. As regards smoking, there seemed to be no marked differences between patients and controls, of either sex. No significant differences were observed in vegetable intake between colorectal adenocarcinoma patients and controls, or between men

Table 1 Main demographic data of the enrolled subjects

\begin{tabular}{lllll}
\hline Demographic data & \multicolumn{2}{l}{ Patients (100) } & \multicolumn{2}{l}{ Control subjects (112) } \\
\hline Gender & Female & Male & Female & Male \\
& 42 & 58 & 56 & 56 \\
Age [y]: median (range) & $65(28-88)$ & $65(46-88)$ & $56(19-78)$ & $57(23-82)$ \\
BMI [kg/m²] & & & & \\
$<18.50$ & $1(2.4 \%)$ & $2(3.4 \%)$ & $3(5.4 \%)$ & $0(0 \%)$ \\
$18.50-24.99$ & $25(59.5 \%)$ & $23(39.7 \%)$ & $37(66.1 \%)$ & $31(55.4 \%)$ \\
$25.00-29.99$ & $12(28.6 \%)$ & $26(44.8 \%)$ & $13(23.2 \%)$ & $18(32.1 \%)$ \\
$\geq 30.00$ & $4(9.5 \%)$ & $7(12.1 \%)$ & $3(5.4 \%)$ & $7(12.5 \%)$ \\
Alcohol consumption $[$ drinks/day] & & & \\
none & $19(45.2 \%)$ & $6(10.3 \%)$ & $32(57.1 \%)$ & $26(46.4 \%)$ \\
$<1$ & $13(31.0 \%)$ & $17(29.3 \%)$ & $13(23.2 \%)$ & $11(19.6 \%)$ \\
$\geq 1$ & $10(23.8 \%)$ & $35(60.4 \%)$ & $11(19.6 \%)$ & $19(34.0 \%)$
\end{tabular}

Red meat intake [portions/week]

$\begin{array}{lllll}\text { none } & 1(2.4 \%) & 0(0 \%) & 3(5.4 \%) & 1(1.8 \%) \\ 1-2 & 15(35.7 \%) & 13(22.4 \%) & 27(48.2 \%) & 29(51.8 \%) \\ 3-4 & 19(45.2 \%) & 35(60.4 \%) & 23(41.1 \%) & 19(33.9 \%) \\ >4 & 7(16.7 \%) & 10(17.2 \%) & 3(5.4 \%) & 7(12.5 \%)\end{array}$

Vegetables [portions/day]

$\begin{array}{lllll}\text { none } & 10(23.8 \%) & 4(6.9 \%) & 10(17.9 \%) & 14(25.0 \%) \\ 1 & 28(66.7 \%) & 51(87.9 \%) & 33(58.9 \%) & 30(53.6 \%) \\ >1 & 4(9.5 \%) & 3(5.2 \%) & 13(23.2 \%) & 12(21.4 \%)\end{array}$

Smoking

never smoker $\quad 28(66.7 \%) \quad 30(51.7 \%) \quad 38(67.9 \%) \quad 30(53.6 \%)$

former smoker $\quad 5(11.9 \%) \quad 17(29.3 \%) \quad 7(12.5 \%) \quad 14(25.0 \%)$

current smoker $\quad 9(21.4 \%) \quad 11(19.0 \%) \quad 11(19.6 \%) \quad 12(21.4 \%)$

Family history of CRC

$\begin{array}{lllll}\text { yes } & 3(7.1 \%) & 11(19.0 \%) & 26(46.4 \%) & 20(35.7 \%)\end{array}$

no $\quad 39(92.9 \%) \quad 47(81.0 \%) \quad 30(53.6 \%) \quad 36(64.3 \%)$

$B M I$ body mass index 
and women. Finally, a family history of CRC was definitely more frequent among controls than cases. The controls in our study were in fact pre-selected subjects at risk for whom a colonoscopy had been prescribed, while in the patients the disease had actually manifested itself.

\section{Fluorescence measurements}

As found in our previous studies [14, 15], the plasma fluorescence emission peaks between 610 and $650 \mathrm{~nm}$ of colorectal adenocarcinoma patients and controls differed significantly. The average IF-INT for all patients was significantly higher than the average for all controls: $170.36 \pm 58.42$ a.u. vs $107.85 \pm 34.55$ a.u. with $P<0.0001$. The Spearman's rho correlation coefficient between IF-INT and TNM staging was $0.224(P=0.011)$, suggesting that there is a weak positive linear correlation between IF-INT and disease severity. With the arbitrary cut-off of 125.00 a.u., the overall accuracy of IF-INT in correctly distinguishing between colorectal adenocarcinoma patients and controls was $84 \%$, with $85 \%$ sensitivity and $79 \%$ specificity. The AUROC was 0.786 .

\section{Artificial neural network}

The optimized ANN architecture was achieved using five hidden neurons; the best validation was reached after 157 iterations, with MSE 0.079 and a coefficient of determination $\mathrm{R}^{2}$ of 0.987 . Table 2 reports the sensitivity, specificity, NPV and PPV with an arbitrary cut-off of 0.60 on ANN output, for the training, validation and test sets. Figure 1 shows the ROC curve for the test set; the resulting AUROC was 0.828 . Table 3 reports the relative importance and rank of the input variables.

\section{Qualitative mass-spectrometric analysis}

Table 4 reports the chromatographic and spectrometric properties of the five porphyrin compounds mainly involved in heme biosynthesis, used to verify their presence in the plasma samples submitted to fluorescence analysis. Theoretical $\mathrm{M} / \mathrm{Z}$ ratios are shown, with the experimental $M / Z$ ratios and the RTs from the analytical method.

Table 2 Performance of the artificial neural network classifier in differential diagnosis of colorectal adenocarcinoma in patients and controls

\begin{tabular}{llll}
\hline & Training set & Validation set & Test set \\
\hline Total population & 146 & 33 & 33 \\
Sensitivity & $88.9 \%$ & $84.6 \%$ & $86.7 \%$ \\
Specificity & $91.9 \%$ & $85.0 \%$ & $88.9 \%$ \\
NPV & $89.5 \%$ & $89.5 \%$ & $88.9 \%$ \\
PPV & $91.4 \%$ & $78.6 \%$ & $86.7 \%$ \\
\hline
\end{tabular}

NPV Negative Predictive Value, PPV Positive Predictive Value

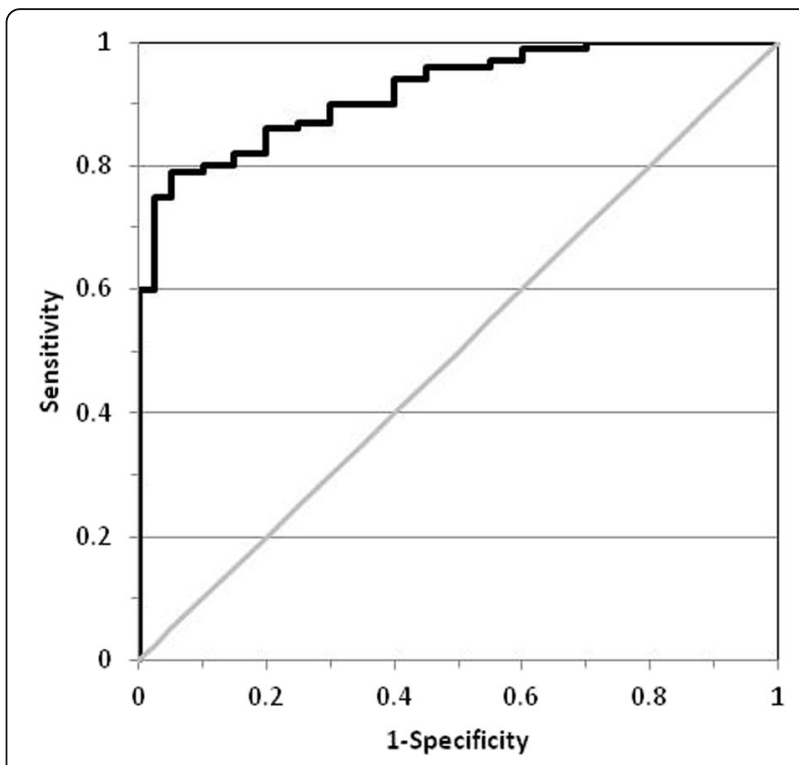

Fig. 1 Receiver operating characteristic curve for the optimized artificial neural network classifier based on demographic data and the integral of the fluorescence emission peak in the range 610$650 \mathrm{~nm}$. The area under the ROC curve was 0.828

Figure 2 shows typical chromatograms (relative abundance vs retention time) of the extracted exact mass of the analytes: A, porphyrin standard solution (reference chromatogram); B, plasma sample of a control subject with low fluorescence emission in the range 610$650 \mathrm{~nm}$; C, plasma sample of a CRC patient with high fluorescence emission in the same range. The peaks shown in Fig. 2 with a non-tabulated RT correspond to substances not contained in the porphyrin standard solution. Verification of the cross-correspondence of the $\mathrm{M} / \mathrm{Z}$ values and RTs enabled us to identify the analytes responsible for the native fluorescence of plasma. Protoporphyrin IX and coproporphyrin I were the only analytes present only in the patients' plasma samples and not in those of the controls. The other analytes were non-specific: biliverdin and iron protoporphyrin IX were present in the plasma samples of both patients and

Table 3 Relative importance and rank of the input variables of the artificial neural network classifier

\begin{tabular}{lll}
\hline & Relative importance & Rank \\
\hline IF-INT & $78.73 \%$ & 1 \\
BMI & $10.22 \%$ & 2 \\
Family history for CRC & $5.01 \%$ & 3 \\
Red meat intake & $4.04 \%$ & 4 \\
Alcohol consumption & $1.30 \%$ & 5 \\
Smoking & $0.45 \%$ & 6 \\
Vegetable intake & $0.25 \%$ & 7 \\
\hline
\end{tabular}

$B M I$ body mass index 
Table 4 Chromatographic and spectrometric properties of the analytes involved in heme biosynthesis

\begin{tabular}{lllll}
\hline Porphyrin & Molecular formula & Theoretical M/Z ratio $[\mathrm{M}+\mathrm{H}]^{+}$ & Experimental $\mathrm{M} / \mathrm{Z}$ ratio $[\mathrm{M}+\mathrm{H}]^{+}$ & $\mathrm{RT}[\mathrm{min}]$ \\
\hline Protoporphyrin IX & $\mathrm{C}_{34} \mathrm{H}_{34} \mathrm{~N}_{4} \mathrm{O}_{4}$ & 563.2653 & 563.2651 & 6.88 \\
Iron protoporphyrin IX & $\mathrm{C}_{34} \mathrm{H}_{32} \mathrm{FeN}_{4} \mathrm{O}_{4}$ & 616.1768 & 616.1775 & 4.48 \\
Biliverdin & $\mathrm{C}_{33} \mathrm{H}_{34} \mathrm{~N}_{4} \mathrm{O}_{6}$ & 583.2551 & 583.2565 & 2.96 \\
Coproporphyrin I & $\mathrm{C}_{36} \mathrm{H}_{38} \mathrm{~N}_{4} \mathrm{O}_{8}$ & 655.2762 & 655.2775 & 1.85 \\
Protoporphyrin IX dimethylester & $\mathrm{C}_{36} \mathrm{H}_{38} \mathrm{~N}_{4} \mathrm{O}_{4}$ & 591.2966 & 591.2961 & 8.06 \\
\hline
\end{tabular}

M/Z Mass to charge ratio, $R T$ Retention time

controls, while protoporphyrin IX dimethylester was not found in either case.

\section{Discussion}

In tumor cells the synthesis of PpIX is highly activated and ferrochelatase mRNA expression is down-regulated so PpIX tends to accumulate specifically in tumor tissues
[27, 28]. Treatment with 5-aminolevulinic acid (5-ALA) results in progressive accumulation of PpIX in malignant tissue but not in the surrounding tissue, thus offering a means of distinguishing healthy from pathological tissues, exploiting the fluorescence properties of PpIX [29]. Several studies have demonstrated the broad applicability in cancer detection of fluorescence analysis of intrinsic or

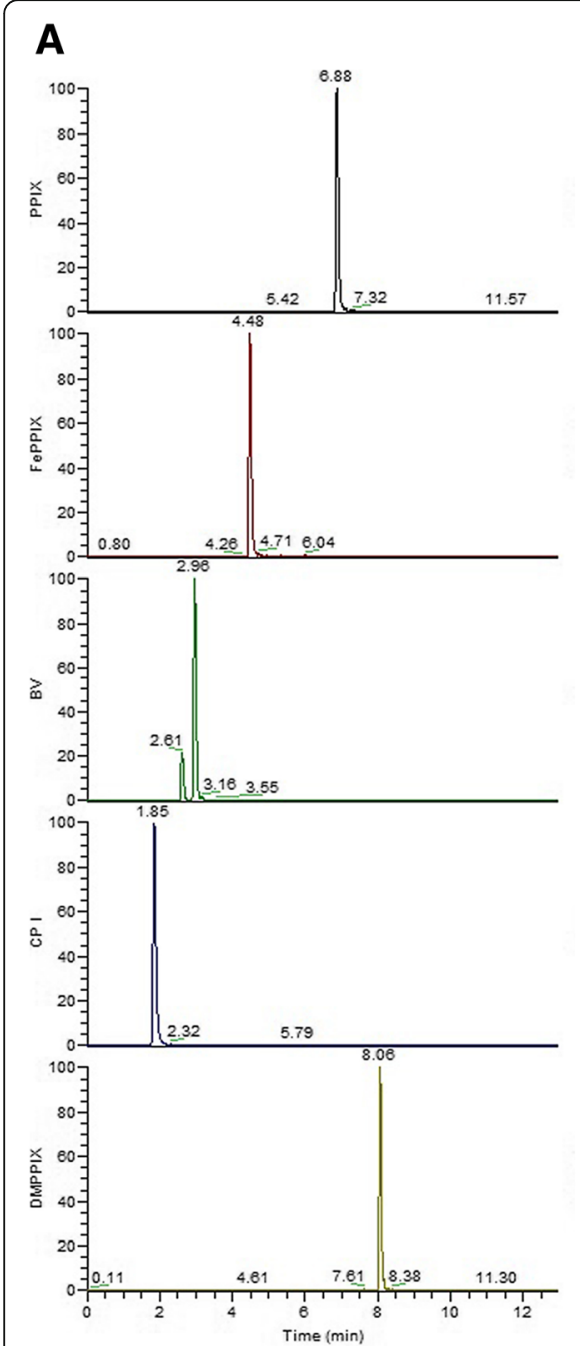

B

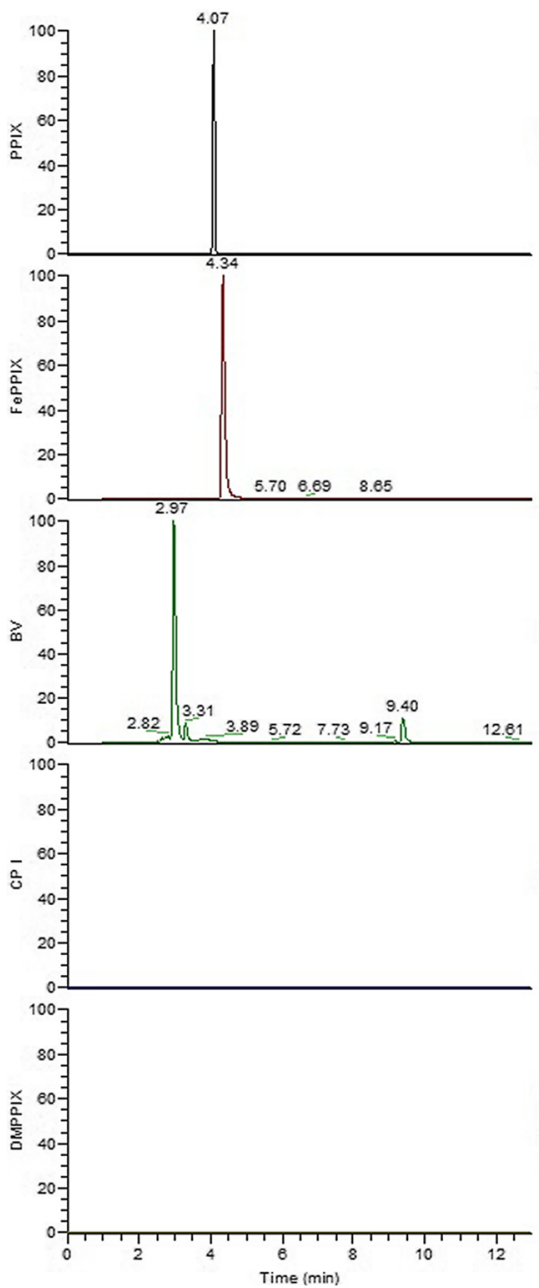

C

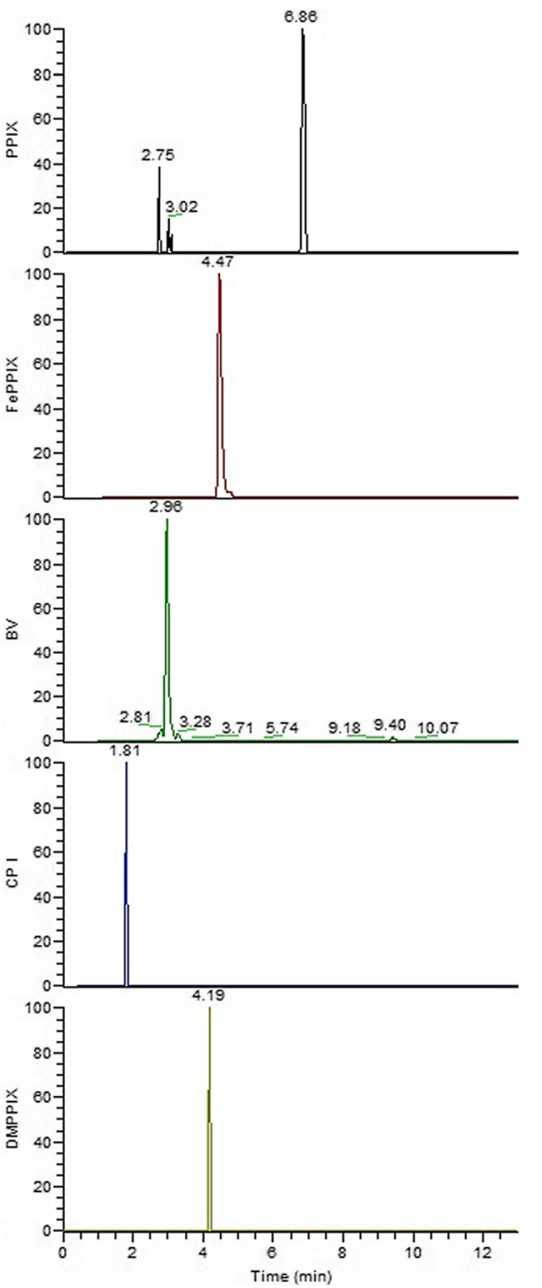

Fig. 2 LC-HRMS chromatograms of: (a) standard solution of protoporphyrin IX (PpIX), iron protoporphyrin IX (FePplX), biliverdin (Bv), coproporphyrin I (Cpl) and protoporphyrin IX dimethyl ester (dmPpIX); (b) plasma sample of a control subject with low fluorescence emission in the range $610-650 \mathrm{~nm}$; (c) plasma sample of a colorectal adenocarcinoma patient with high fluorescence emission in the range 610-650 $\mathrm{nm}$ 
stimulated PpIX, on samples of ex vivo tissue and biofluids in which the PpIX may pour out of the tumor cells $[21-25,30]$.

In this study we examined the potential utility of the plasma concentrations of intrinsic porphyrin compounds in the early diagnosis of colorectal adenocarcinoma. We found significantly higher endogenous porphyrin concentrations in the plasma of colorectal adenocarcinoma patients than control subjects; this was indirectly evident from analysis of the native fluorescence spectrum and directly confirmed by LC-HRMS analysis. Fluorescence analysis is a very sensitive technique, easy and quick to implement but it cannot precisely determine the agent responsible for the fluorimetric signal, since different compounds may have the same fluorescence spectrum. LC-HRMS is much more laborious and expensive but does determine the agents responsible for the fluorescence signal by calculating the mass of the substances in the sample.

In the presented method, the analytical descriptor selected to distinguish between colorectal adenocarcinoma patients and control subjects is the integral of the fluorescence emission peak between $610 \mathrm{~nm}$ and $650 \mathrm{~nm}$, acquired with a conventional fluorimeter following blue light irradiation. According to our LC-HRMS analysis, the agents responsible for plasma fluorescence emission in the selected wavelength range were mainly protoporphyrin-IX and coproporphyrin-I. Both these compounds have fluorescence emission in the range $610-650 \mathrm{~nm}$ when excited at $405 \mathrm{~nm}$, with maximum emission wavelengths at respectively 622 and $632 \mathrm{~nm}$ [31]. Investigations currently in progress might clarify whether one or both porphyrins are accumulated by cancer cells more than by healthy ones.

About $75 \%$ of CRC patients have sporadic forms of the disease. The remaining $25 \%$ have a family history of colorectal cancer, adenomatous polyps or inherited syndromes such as familial adenomatous polyposis (FAP) and Lynch syndrome, suggesting a contribution of inherited genes, shared environmental factors, or some combination of these [1, 4, 32]. Other CRC risk factors include overweight, especially having a larger waistline, type 2 diabetes, physical inactivity, a diet that is high in red and processed meats and poor in vegetables and fruits, smoking, heavy alcohol use, older age, a personal history of adenomatous polyps and inflammatory bowel disease, including ulcerative colitis or Crohn's disease [4, 32-34]. A mathematical predictive model that integrates risk factors and the result of one or more tumor markers could potentially enhance the diagnostic performance of the markers themselves by simulating the diagnostic process of a physician who simultaneously evaluates the results of laboratory tests and the patient's personal and family medical history. ANNs offer a relatively new method for predictive modeling in medicine, and are used to map and predict outcomes in complex relationships between given 'inputs' (e.g. risk factors, laboratory test results, morphological findings from radiological examinations) and sought-after 'outputs' (classification or diagnosis) [17, 35-37]. In contrast with traditional statistical techniques, ANNs are capable of automatically resolving these relationships without the need for a priori assumptions about the nature of the interactions between variables; they employ various statistical, probabilistic and optimization techniques that enable computers to learn from examples and to detect hard-to-discern patterns from large, noisy or complex data sets. The ANN model we describe is a clinical decision support tool based on plasma porphyrins accumulation and risk factors. In this preliminary study, the pre-selection of the enrolled subjects did not permit us to consider some risk factors for this pathology, for example age. Furthermore, the personal history of chronic gastrointestinal diseases was not taken into consideration because we did not know a priori how these pathologies might interact with the plasma native fluorescence spectrum. Our future efforts will focus on extending the study to a larger cohort of subjects in which to consider all the possible risk factors.

We found the overall accuracy of IF-INT for colorectal adenocarcinoma detection was $84 \%$, with $85 \%$ sensitivity and $79 \%$ specificity, while the overall accuracy of the ANN model (IF-INT plus risk factors) was $88 \%$, with $87 \%$ sensitivity and $90 \%$ specificity. Although the relative importance of each individual risk factor is significantly lower than that of porphyrin concentration, the contribution of risk factors is not irrelevant in the diagnostic performance of the ANN model and could hopefully be increased considering other risk factors currently unverifiable on the our study population. Accuracy, sensitivity and specificity of our ANN model are comparable to the performances of FITs for colorectal cancer detection, as reported in a meta-analysis performed by Lee et al. [38] that suggests that the pooled sensitivity and specificity of FITs were approximately $79 \%$ and $94 \%$, respectively, with an overall accuracy of $95 \%$. FIT sensitivity may decrease with any delay in processing the sample and, like for all stool-based tests, compliance is lower than with blood-based analysis. To appreciate the actual predictive performances of IF-INT and of our ANN model in comparison to FIT, we are planning a comparative study using colonoscopy as the gold standard to assess the two markers on the same population of colorectal adenocarcinoma patients and control subjects.

\section{Conclusion}

The results reported in our paper confirm the presence of diagnostic levels of endogenous porphyrin compounds in the blood plasma of colorectal adenocarcinoma patients 
and suggest that the measurement of plasma porphyrins concentration may be applied, together with risk factors, as a clinical decision support tool for the early detection of colorectal adenocarcinoma. Further investigations of selected CRC patients are under way to assess IF-INT's utility as a marker that correlates with survival and response to therapy.

\section{Abbreviations}

ANN: Artificial neural network; AUROC: Area under receiver operating characteristic curve; BMI: Body mass index; Bv: Biliverdin;

Cpl: Coproporphyrin-l; CRC: Colorectal cancer; dmPpIX: Protoporphyrin IX dimethyl ester; FePpIX: Iron protoporphyrin IX; FIT: Fecal immunochemical test; FS: Full scan; FWHM: Full width at half-maximum; gFOBT: Guaiac-based fecal occult blood test; IF-INT: Integral of the fluorescence emission peak; LCHRMS: Liquid chromatography-high resolution mass spectrometry; MSE: Mean square error; NPV: Negative predictive value; PpIX: Protoporphyrin-IX; PPV: Positive predictive value; ROC: Receiver operating characteristic curve; RT: Retention time

\section{Acknowledgments}

The authors would like to thank Milena Sant, Elisabetta Meneghini, Pamela Minicozzi and Claudia Vener of the Analytical Epidemiology and Health Impact Unit Fondazione IRCCS Istituto Nazionale dei Tumori (Milan, Italy) for their contribution in correcting the manuscript.

\section{Availability of data and materials}

The datasets generated and analyzed during the current study are available from the corresponding author on reasonable request.

\section{Authors' contributions}

$M L, A d C, E P$ and ES conceived the project and performed the experimental design. LB, DM and EL provided clinical samples and aided in clinical interpretation. ML, AdC, ES, GG and AC executed experimental measurements and data analysis. ML, AdC and GG wrote the manuscript. All of the authors read and approved the final version of this manuscript.

\section{Ethics approval and consent to participate}

Approval for the study was obtained from Ethics Committee of the Fondazione IRCCS Istituto Nazionale dei Tumori, Milan, Italy. All participants provided their written informed consent to participate in this study.

\section{Consent for publication}

Not applicable.

\section{Competing interests}

The authors declare that they have no competing interests.

\section{Publisher's Note}

Springer Nature remains neutral with regard to jurisdictional claims in published maps and institutional affiliations.

\section{Author details}

${ }^{1}$ Medical Physics Unit, Fondazione IRCCS Istituto Nazionale dei Tumori, Via Venezian 1, 20133 Milan, Italy. ${ }^{2}$ Epidemiology and Prevention Unit, Fondazione IRCCS Istituto Nazionale dei Tumori, Milan, Italy. ${ }^{3}$ Colorectal Cancer Unit, Fondazione IRCCS Istituto Nazionale dei Tumori, Milan, Italy. ${ }^{4}$ Health Administration, Fondazione IRCCS Istituto Nazionale dei Tumori, Milan, Italy. ${ }^{5}$ Department of Pathology and Laboratory Medicine, Fondazione IRCCS Istituto Nazionale dei Tumori, Milan, Italy. ${ }^{6}$ Department of Medical Oncology, Fondazione IRCCS Istituto Nazionale dei Tumori, Milan, Italy.

Received: 14 February 2018 Accepted: 16 August 2018

Published online: 22 August 2018

\section{References}

1. Siegel RL, Miller KD, Fedewa SA, et al. Colorectal Cancer statistics, 2017. CA Cancer J Clin. 2017;67:177-93.
2. Schoen RE, Pinsky PF, Weissfeld $J$, et al. Colorectal-cancer incidence and mortality with screening flexible sigmoidoscopy. N Engl J Med. 2012;366: 2345-57.

3. Zauber AG, Winawer SJ, O'Brien MJ, et al. Colonoscopic polypectomy and long-term prevention of colorectal-cancer deaths. N Engl J Med. 2012;366: 687-96.

4. American Cancer Society. Colorectal Cancer Facts \& Figures 2017-2019. Atlanta: American Cancer Society. 2017:

5. El Zoghbi M, Cummings LC. New era of colorectal cancer screening. World J Gastrointest Endosc. 2016:8(5):252-8.

6. Imperiale TF, Ransohoff DF, Itzkowitz SH, et al. Multitarget stool DNA testing for colorectal-cancer screening. N Engl J Med. 2014;370:1287-97.

7. Shastri YM, Loitsch S, Hoepffner N, et al. Comparison of an established simple office-based immunological FOBT with fecal tumor pyruvate kinase type M2 (M2-PK) for colorectal cancer screening: prospective multicenter study. Am J Gastroenterol. 2008;103:1496-504.

8. Adler A, Geiger S, Keil A, et al. Improving compliance to colorectal cancer screening using blood and stool based tests in patients refusing screening colonoscopy in Germany. BMC Gastroenterol. 2014;14:183.

9. Negm OH, Hamed MR, Schoen RE, et al. Human blood autoantibodies in the detection of colorectal cancer. PLoS One. 2016;11(7):e0156971.

10. Poh CF, Anderson DW, Durham JS, et al. Fluorescence visualization-guided surgery for early-stage oral Cancer. JAMA Otolaryngol Head Ne. 2016;142(3): 209-16.

11. Jing $Y$, Wang $Y$, Wang $X$, et al. Label-free imaging and spectroscopy for early detection of cervical cancer. J. Biophotonics. 2018:e-201700245.

12. $\mathrm{Xu} \mathrm{HN}$, Tchou J, Feng $M$, et al. Optical redox imaging indices discriminate human breast cancer from normal tissues. J Biomed Opt. 2016;21(11): 114003.

13. Bottiroli G, Croce AC, Locatelli D, et al. Natural fluorescence of normal and neoplastic human colon: a comprehensive "ex vivo" study. Lasers Surg Med. 1995;16:48-60.

14. Lualdi M, Colombo A, Leo E, et al. Natural fluorescence spectroscopy of human blood plasma in the diagnosis of colorectal cancer: feasibility study and preliminary results. Tumori. 2007;93:567-71.

15. Lualdi M, Battaglia L, Colombo A, et al. Colorectal cancer detection by means of optical fluoroscopy. A study on 494 subjects. Front Biol. 2010;E2: 694-700.

16. International Union Against Cancer (UICC) TNM Classification of Malignant Tumours, 7th ed. 2009 J D Brierley, M K Gospodarowicz, C Wittekind eds. New York: Wiley-Blackwell.

17. Amato F, López A, Peña-Méndez EM, et al. Artificial neural networks in medical diagnosis. J Appl Biomed. 2013;11:47-58.

18. Haupt SE, Pasini A, Marzban C. Artificial intelligence methods in the environmental sciences. New York: springer; 2009. 424

19. Pasini A. Artificial neural networks for small dataset analysis. J Thorac Dis. 2015:7(5):953-60

20. Ibrahim OM. A comparison of methods for assessing the relative importance of input variables in artificial neural networks. J Appl Sci Res. 2013;9(11):5692-700.

21. Masilamani V, Al-Zhrani K, Al-Salhi M, et al. Cancer diagnosis by autofluorescence of blood components. J Luminescence. 2004;109(3-4): $143-54$

22. Courrol LC, de Oliveira Silva FR, Coutinho EL, et al. Study of blood porphyrin spectral profile for diagnosis of tumor progression. J Fluoresc. 2007;17:289-92.

23. Kalaivani R, Masilamani $V$, Sivaji K, et al. Fluorescence spectra of blood components for breast cancer diagnosis. Photomed Laser Surg. 2008;26(3): 251-6.

24. de Oliveira Silva FR, Bellini MH, Tristão VR, et al. Intrinsic fluorescence of protoporphyrin IX from blood samples can yield information on the growth of prostate tumors. J Fluoresc. 2010;20:1159-65.

25. Ota U, Fukuhara H, Ishizuka M, et al. Plasma protoporphyrin IX following administration of 5-aminolevulinic acid as a potential tumor marker. Mol Clin Oncol. 2015;3(4):797-801.

26. Physical Status WHO. The use and interpretation of anthropometry: report of a World Health Organization (WHO) expert committee. Geneva. Switzerland: World Health Organization; 1995.

27. Kemmner W, Wan K, Ruttinger $S$, et al. Silencing of human ferrochelatase causes abundant protoporphyrin-IX accumulation in colon cancer. FASEB J. 2008:22:500-9. 
28. Sachar M, Anderson KE, Ma X. Protoporphyrin IX: the good, the bad, and the ugly. J Pharmacol Exp Ther. 2016;356(2):267-75.

29. Yang X, Li W, Palasuberniam P, et al. Effects of silencing heme biosynthesis enzymes of 5-aminolevulinic acid-mediated protoporphyrin IX fluorescence and photodynamic therapy. Photochem Photobiol. 2015;91:923-30.

30. Nakamura M, Nishikawa J, Hamabe K, et al. Preliminary study of photodynamic diagnosis using 5-aminolevulinic acid in gastric and colorectal tumors. World J Gastroenterol. 2015;21:6706-12.

31. Berezin MY, Achilefu S. Fluorescence lifetime measurements and biological imaging. Chem Rev. 2010;110(5):2641-84.

32. Migliore L, Migheli F, Spisni R, Coppede F. Genetics, cytogenetics, and epigenetics of colorectal cancer. J Biomed Biotech. 2011;2011:792362. https://doi.org/10.1155/2011/792362.

33. Keimling M, Renehan AG, Behrens $G$, et al. Comparison of associations of body mass index, abdominal adiposity and risk of colorectal cancer in a large prospective cohort study. Cancer Epidemiol Biomark Prev. 2013;22(8): 1383-94.

34. Baena R, Salinas P. Diet and colorectal cancer. Maturitas. 2015;80:258-64

35. Hu X, Cammann H, Meyer HA, et al. Artificial neural networks and prostate cancer-tools for diagnosis and management. Nat Rev Urol. 2013;10:17482.

36. Muralidaran C, Dey P, Nijhawan R, et al. Artificial neural network in diagnosis of urothelial cell carcinoma in urine cytology. Diagn Cytopathol. 2015;43(6): 443-9.

37. Aguiar FS, Torres RC, Pinto JVF, et al. Development of two artificial neural network models to support the diagnosis of pulmonary tuberculosis in hospitalized patients in Rio de Janeiro, Brazil. Med Biol Eng Comput. 2016; 54:1751-9.

38. Lee JK, Liles EG, Bent S, et al. Accuracy of fecal immunochemical tests for colorectal cancer: systematic review and meta-analysis. Ann Intern Med. 2014;160(3):171.

Ready to submit your research? Choose BMC and benefit from:

- fast, convenient online submission

- thorough peer review by experienced researchers in your field

- rapid publication on acceptance

- support for research data, including large and complex data types

- gold Open Access which fosters wider collaboration and increased citations

- maximum visibility for your research: over $100 \mathrm{M}$ website views per year

At $\mathrm{BMC}$, research is always in progress.

Learn more biomedcentral.com/submissions 